\title{
Anode-pore tortuosity in solid oxide fuel cells found from gas and current flow rates
}

\section{Authors: V. Hugo Schmidt and Chih-Long Tsai}

NOTICE: this is the author's version of a work that was accepted for publication in Journal of Power Sources. Changes resulting from the publishing process, such as peer review, editing, corrections, structural formatting, and other quality control mechanisms may not be reflected in this document. Changes may have been made to this work since it was submitted for publication. A definitive version was subsequently published in Journal of Power Sources, VOL\# 180, ISSUE\# 1, (2008), DOI\# 10.1016/j.jpowsour.2008.01.073.

V. Hugo Schmidt and Chih-Long Tsai, "Anode-pore tortuosity in solid oxide fuel cells found from gas and current flow rates," Journal of Power Sources 180, 253-264 (2008). doi: 10.1016/ j.jpowsour.2008.01.073.

Made available through Montana State University's ScholarWorks 


\title{
Anode-pore tortuosity in solid oxide fuel cells found from gas and current flow rates
}

\author{
V. Hugo Schmidt*, Chih-Long Tsai \\ Department of Physics, EPS 264, Montana State University, Bozeman, MT 59717, USA \\ Received 7 December 2007; received in revised form 18 January 2008; accepted 22 January 2008 \\ Available online 12 February 2008
}

\begin{abstract}
The effect of solid oxide fuel cell (SOFC) anode thickness, porosity, pore size, and pore tortuosity on fuel and exhaust gas flow is calculated. Also determined is the concentration of these gases and of diluent gases as a function of position across the anode. The calculation is based on the dusty-gas model which includes a Knudsen (molecule-wall) collision term in the Stefan-Maxwell equation which is based on unlike-molecule collisions. Commonly made approximations are avoided in order to obtain more exact results. One such approximation is the assumption of uniform total gas pressure across the anode. Another such approximation is the assumption of zero fuel gas concentration at the anode-electrolyte interface under the anode saturation condition for which the SOFC output voltage goes to zero. Elimination of this approximation requires use of a model we developed (published elsewhere) for terminal voltage $V$ as a function of electrolyte current density $i$. Key formulae from this model are presented. The formulae developed herein for gas flow and tortuosity are applied to the results of a series of careful experiments performed by another group, who used binary and ternary gas mixtures on the anode side of an SOFC. Our values for tortuosity are in a physically reasonable low range, from 1.7 to 3.3. They are in fair agreement with those obtained by the other group, once a difference in nomenclature is taken into account. This difference consists in their definition of tortuosity being what some call tortuosity factor, which is the square of what we and some others call tortuosity. The results emphasize the need for careful design of anode pore structures, especially in anode-supported SOFCs which require thicker anodes.
\end{abstract}

(C) 2008 Elsevier B.V. All rights reserved.

Keywords: Solid oxide fuel cells; Anode-supported; Tortuosity; Dusty-gas model; Gas flow

\section{Introduction}

Solid oxide fuel cells (SOFCs) provide efficient generation of electricity using a variety of fuels. They produce only steam exhaust if hydrogen is the fuel. The same device can act as a steam electrolyzer to produce hydrogen and oxygen from steam if a dc voltage is applied.

Present SOFCs operate in the $800-1000{ }^{\circ} \mathrm{C}(1073-1273 \mathrm{~K})$ range, but strong efforts are in progress to extend this range downward to $600{ }^{\circ} \mathrm{C}(873 \mathrm{~K})$ or lower to minimize interconnect cost by allowing use of stainless steel, and to reduce problems associated with thermal expansion, atomic migration, and corrosion. The main obstacle for this temperature reduction is the rapid decrease in solid electrolyte oxygen ion conductivity with decreasing temperature. Accordingly, the electrolyte is made as thin as possible, of order $10 \mu \mathrm{m}$. This requires that an electrode layer, usually the anode, must be made thick enough to mechanically support the cell.

With a thicker anode, one must calculate carefully the fuel and exhaust gas concentrations at the anode-electrolyte interface. These concentrations (or corresponding partial pressures) strongly affect $V(i)$, the terminal voltage $V$ as a function of electrolyte current density $i$. The anode characteristics (assuming a much thinner cathode) also under some conditions determine the maximum attainable $i$. This is the current density when the fuel flow rate is high enough to reduce the fuel gas partial pressure at the interface to near zero, and the SOFC output voltage to exactly zero. This fuel pressure gradient in the anode is increased by the inflowing fuel molecule collisions with the outflowing exhaust molecules. 
Table 1

Tortuosities calculated for $\mathrm{H}_{2} / \mathrm{H}_{2} \mathrm{O}$ and $\mathrm{CO} / \mathrm{CO}_{2}$ binary systems

\begin{tabular}{|c|c|c|c|c|c|c|}
\hline Fuel & p.p. (atm) & $i_{\mathrm{as}}\left(\mathrm{Acm}^{-2}\right)$ & $\tau$ & $\tau\left(n_{\mathrm{fas}}=0\right)$ & $\left(\tau_{\mathrm{JV}}\right)^{1 / 2}$ & $\tau_{\mathrm{JV}}$ \\
\hline $\mathrm{H}_{2}$ & 0.2 & 1.3 & 1.945 & 2.179 & 2.211 & 4.89 \\
\hline $\mathrm{H}_{2}$ & 0.34 & 2.1 & 2.024 & 2.243 & 2.294 & 5.26 \\
\hline $\mathrm{H}_{2}$ & 0.5 & 3.25 & 1.913 & 2.136 & 2.211 & 4.89 \\
\hline $\mathrm{CO}$ & 0.18 & 0.21 & 2.730 & 2.871 & 2.876 & 8.27 \\
\hline $\mathrm{CO}$ & 0.23 & 0.31 & 2.502 & 2.656 & 2.661 & 7.08 \\
\hline $\mathrm{CO}$ & 0.32 & 0.49 & 2.311 & 2.475 & 2.484 & 6.17 \\
\hline $\mathrm{CO}$ & 0.44 & 0.73 & 2.194 & 2.366 & 2.377 & 5.65 \\
\hline
\end{tabular}

Fuel partial pressures (p.p.), anode saturation current densities $\left(i_{\text {as }}\right)$, and $\tau_{\mathrm{JV}}$ are from JV Table 2 [1].

Table 2

Tortuosities calculated for various ternary system diluent gases and partial pressures (p.p. in atm), and measured anode saturation current densities $\left(i_{\text {as }}\right.$, A cm ${ }^{-2}$ )

\begin{tabular}{|c|c|c|c|c|c|c|c|}
\hline Diluent & p.p. & $i_{\mathrm{as}}$ & $\tau_{\operatorname{lin}}$ & $\tau_{\text {quad }}$ & $\tau_{\text {exact }}$ & $\left(\tau_{\mathrm{JV}}\right)^{1 / 2}$ & $\tau_{\mathrm{JV}}$ \\
\hline $\mathrm{He}$ & 0.78 & 1.5 & 1.7159 & 1.7025 & 1.7025 & 2.3707 & 5.62 \\
\hline $\mathrm{He}$ & 0.65 & 2.4 & 1.7295 & 1.7069 & 1.7068 & 2.3130 & 5.35 \\
\hline $\mathrm{He}$ & 0.53 & 3.25 & 1.7371 & 1.7052 & 1.7051 & 2.2583 & 5.1 \\
\hline $\mathrm{He}$ & 0.42 & 4 & 1.7634 & 1.7215 & 1.7214 & 2.2271 & 4.96 \\
\hline $\mathrm{N}_{2}$ & 0.8 & 0.89 & 2.8705 & 2.8020 & 2.8029 & 2.7495 & 7.56 \\
\hline $\mathrm{N}_{2}$ & 0.67 & 1.56 & 2.7149 & 2.6225 & 2.6240 & 2.6627 & 7.09 \\
\hline $\mathrm{N}_{2}$ & 0.57 & 2.3 & 2.4401 & 2.3469 & 2.3484 & 2.4597 & 6.05 \\
\hline $\mathrm{N}_{2}$ & 0.5 & 2.65 & 2.4467 & 2.3433 & 2.3450 & 2.4860 & 6.18 \\
\hline $\mathrm{CO}_{2}$ & 0.81 & 0.7 & 3.4465 & 3.3183 & 3.3225 & 3.0000 & 9 \\
\hline $\mathrm{CO}_{2}$ & 0.68 & 1.43 & 2.9452 & 2.8038 & 2.8094 & 2.6833 & 7.2 \\
\hline $\mathrm{CO}_{2}$ & 0.6 & 1.88 & 2.8036 & 2.6560 & 2.6622 & 2.6153 & 6.84 \\
\hline $\mathrm{CO}_{2}$ & 0.5 & 2.4 & 2.7221 & 2.5663 & 2.5730 & 2.6019 & 6.77 \\
\hline
\end{tabular}

The first three and last columns are from JV [1].

A key parameter in determining the pressure gradient is the tortuosity $\tau$, which is the ratio of the typical diffusion path length to the electrode thickness. Measurement of its value is needed for determining the quality of the anode pore configuration. Also, $\tau$ is a necessary parameter in any model for $V(i)$, and conversely we will show how our model for $V(i)$ provides a more accurate determination of $\tau$.

The paper begins with a description of the Jiang-Virkar [1] experiment to which our analysis is applied. This is followed by a discussion of the Stefan-Maxwell equation including both Knudsen (molecule collisions with pore walls) and binary collision effects. Then the theory and results for binary and ternary anode gas mixtures are presented, followed by conclusions and recommendations.

\section{Description of Jiang-Virkar experiment}

Jiang and Virkar [1] performed a series of careful experiments on flow of various gas mixtures in the anode of an SOFC. This paper will be referred to as JV. JV studied binary system anode-side gas mixtures $\mathrm{H}_{2} / \mathrm{H}_{2} \mathrm{O}$ and $\mathrm{CO} / \mathrm{CO}_{2}$, and ternary systems with incoming $\mathrm{H}_{2}$ fuel diluted with $\mathrm{He}, \mathrm{N}_{2}$, or $\mathrm{CO}_{2}$. In all cases, the total metered inflow rate of the two gases was held at $140 \mathrm{~mL} \mathrm{~min}^{-1}$, corresponding to $6.286 \times 10^{19}$ molec s$^{-1}$ as explained below. The "ternary" systems are called ternary because $\mathrm{H}_{2} \mathrm{O}$ (steam) "exhaust" is produced at the anode-electrolyte interface and mixes with the hydrogen and diluent gas. We compare their calculations of tortuosity $\tau$ with values found from our analysis. We use the definition of tortuosity as the average actual path length traversed through the anode by a fuel or exhaust molecule, divided by the anode thickness. There are other definitions in the literature; in fact, the term "tortuosity" has a "tortuous" history [2]. In particular, JV and some others define tortuosity as what we and some others [2] call "tortuosity factor." The low range of $\tau$ values we obtain, from 1.7 to 3.3 , is in accord with the 2.5-3 range found by Williford et al. [3] who criticized reports of "anomalously high" tortuosities in the 6-17 range.

In the JV experiments, the cathode-side gas was air. Both sides must have been at $1 \mathrm{~atm}$ sea level in order to reproduce their results, even though their laboratory is at the University of Utah, well above sea level. The partial pressures in atmospheres for the various metered input gases are given in our Tables 1 and 2 and Figs. 2 and 3. The temperature $T$ was $800^{\circ} \mathrm{C}=1073 \mathrm{~K}$ for all their experiments. Their SOFC consisted of five layers, as shown in Fig. 1, and constructed of the materials listed below.

Anode

Anode interlayer

Electrolyte

Cathode interlayer

Cathode
$\mathrm{Ni}+$ YSZ (yttria-stabilized zirconia)

$\mathrm{Ni}+\mathrm{YSZ}$

YSZ + SDC (samaria-doped ceria) bilayer

LSC (Sr-doped $\left.\mathrm{LaCoO}_{3}\right)+\mathrm{SDC}$

LSC 


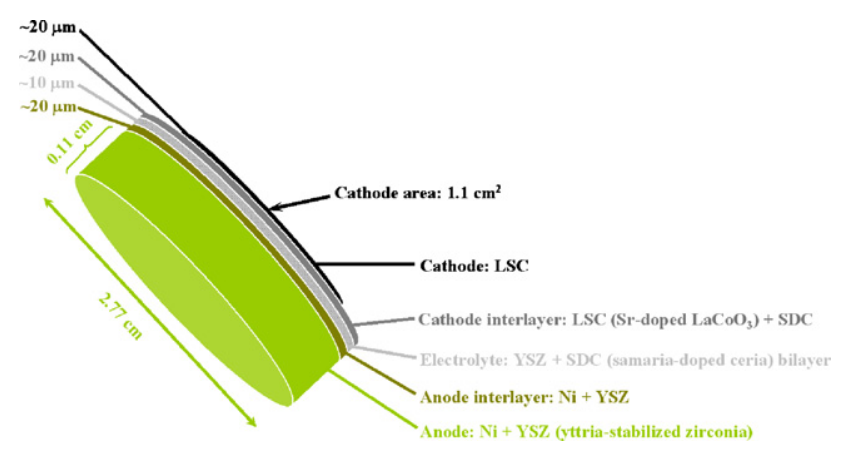

Fig. 1. Drawing of SOFC employed by Jiang and Virkar [1].

The anode thickness $w=0.11 \mathrm{~cm}$, and its porosity $\phi=0.54$. Their estimated pore radius from SEM micrographs was $\sim 0.5 \mu \mathrm{m}$.

\section{Dusty-gas model for gas flow in pores}

The dusty-gas model consists of the Stefan-Maxwell equation including the Knudsen term. In molar units it is [1]

$$
\frac{N_{i}}{D_{\mathrm{K} i}}+\sum_{j \neq i} \frac{X_{j} N_{i}-X_{i} N_{j}}{D_{i j}}=-\frac{(1 / R T) \partial\left(P X_{i}\right)}{\partial x},
$$

where $N_{i}$ and $N_{j}$ are molar fluxes of components $i$ and $j\left(\mathrm{~mol} \mathrm{~cm}^{-2} \mathrm{~s}^{-1}\right)$, respectively, $D_{\mathrm{K} i}$ and $D_{i j}$ are the Knudsen diffusion coefficient for component $i$ and the binary diffusion coefficient for components $i$ and $j$, respectively, $X_{i}$ and $X_{j}$ are the fractional molar concentrations of components $i$ and $j, P$ is the total pressure, $R$ is the gas constant, and $T$ is the absolute temperature. We emphasize that $x$ is the coordinate along a typical diffusion path, not across the anode, so the total diffusion path length is $\tau w$, the product of the tortuosity and the anode thickness. Note that this analysis ignores laminar flow (permeation flux) effects [4] which are considered unimportant in small pores.

Note further that Eq. (1) is modified from Eq. (9) of the JV paper in that $P$ is outside the derivative in their paper. That is correct only in the approximation that $P$ is independent of position. The total pressure of the incoming gases and the gases in the plenums is one atmosphere, denoted as $P_{1}$, but the total pressure $P$ in the anode and cathode pores will be a function of position in the pore. We will show that $P$ varies across the anode by amounts of order $10 \%$.

The Knudsen diffusion coefficient only considers collisions with the wall, and from the kinetic theory of gases is

$$
D_{\mathrm{K}, i}=\frac{2}{3}\left(\frac{8 R T}{\pi M_{i}}\right)^{1 / 2} \bar{r}
$$

where $\bar{r}$ is the mean pore radius $(0.5 \mu \mathrm{m})$ and $M_{i}$ is the molar mass. At $1073 \mathrm{~K}$ the $D_{\mathrm{K} i}$ values for this pore radius are 11.3 and $3.00 \mathrm{~cm}^{2} \mathrm{~s}^{-1}$ for $\mathrm{H}_{2}$ and $\mathrm{CO}$, respectively. To convert Eq. (2) to molecular units, we replace $R / M_{i}$ by $k / m_{i}$, where $k$ is Boltzmann's constant, $1.38 \times 10^{-23} \mathrm{~J} \mathrm{~K}^{-1}$ and $m_{i}$ is molecular mass. We convert to molecular units to make some equations simpler.

The binary diffusion coefficients $D_{i j}$ for various gas-molecule pairs at three temperatures are tabulated in JV Table 1 . The coefficients for the two 2-species cases, $\mathrm{H}_{2} / \mathrm{H}_{2} \mathrm{O}$ and $\mathrm{CO} / \mathrm{CO}_{2}$, have $D_{i j 1}$ values of 7.704 and $1.408 \mathrm{~cm}^{2} \mathrm{~s}^{-1}$, respectively at $1073 \mathrm{~K}$. The subscript 1 indicates that the values are for $1 \mathrm{~atm}$ total pressure.

To convert Eq. (1) to molecular units, several steps are necessary. The $X_{i}$ are dimensionless mole or molecular fractions of the total gas concentration. The $N_{i}$ are converted to $J_{i}$ molecules per unit area and time by multiplying by Avogadro's number $N_{\mathrm{A}}$. The gas constant $R$ has moles in the denominator, so putting $N_{\mathrm{A}}$ in the numerator changes $R$ to $k$. But $P=n k T$, where $n$ is the total number of gas molecules per unit volume, so the $k T$ factors cancel. Since $n X_{i}=n_{i}$, the right side of Eq. (1) is simply $-\partial n_{i} / \partial x$. $D_{i j}$ has the total concentration $n$ in its denominator, but since $D_{i j}$ itself is in the denominator in Eq. (1), $n$ appears in the numerator in that term, and $n$ multiplied by $X_{i}$ or $X_{j}$ changes them to $n_{i}$ or $n_{j}$. Thus, Eq. (1) converts to

$$
\frac{J_{i}}{D_{\mathrm{K} i}}+\frac{\left(J_{i} n_{j}-J_{j} n_{i}\right) k T}{D_{i j 1} P_{1}}=-\frac{\partial n_{i}}{\partial x} .
$$

Here, $D_{i j 1}$ is the binary diffusion coefficient at $P_{1} \equiv 1 \mathrm{~atm}$, and the $n k T$-type terms in the numerator divided by $P_{1}$ convert $D_{i j 1}$ to $D_{i j}$ at the actual total pressure at any position $x$ along the anode pore.

The variable $x$ is distance along the pore, so the total length $L$ of the pore is $L=\tau w$, where $\tau$ is the anode pore tortuosity and $w$ is the $0.11 \mathrm{~cm}$ thickness of the anode. 


\section{Calculation for binary gas input}

For the two binary gas systems $\mathrm{H}_{2} / \mathrm{H}_{2} \mathrm{O}$ and $\mathrm{CO} / \mathrm{CO}_{2}$, if subscript $\mathrm{f}$ is for the fuel gas and e is for the exhaust gas, $J_{\mathrm{e}}=-J_{\mathrm{f}}$ in Eq. (3). Here, $J_{\mathrm{f}}=i \tau / \phi q$, where $i$ is the current density $\left(\mathrm{Acm}^{-2}=\mathrm{C} \mathrm{cm}^{-2} \mathrm{~s}^{-1}\right)$ in the solid electrolyte and $q$ is the charge $\left(3.2 \times 10^{-19} \mathrm{C}_{\text {molecule }}^{-1}\right)$ carried per gas molecule annihilated/created in the reaction at the anode/electrolyte interface.

The $\tau / \phi$ factor is an enhancement factor by which the flow density $J_{i}$ is enhanced compared to its value if the anode were completely porous $(\phi=\tau=1)$. To derive this result, note that this enhancement factor for an anode of area $S$ with $N_{\text {pa }}$ pores each of cross-sectional area $A_{x}$ is $S / N_{\mathrm{pa}} A_{x}$. The porosity $\phi$ is the total pore volume $N_{\mathrm{pa}} A_{x} \tau w$ divided by the anode volume $S w$, so $\phi=N_{\mathrm{pa}} A_{x} \tau / S$. By rearranging this equation we find that the enhancement factor $S / N_{\mathrm{pa}} A_{x}$ is $\tau / \phi$.

Eq. (3) for the fuel gas can now be written, after setting $n_{\mathrm{e}}+n_{\mathrm{f}}=n$, as

$$
\frac{\partial n_{\mathrm{f}}}{\partial x}=-\left(\frac{i \tau}{\phi q}\right)\left(\frac{1}{D_{\mathrm{Kf}}}+\frac{n k T}{D_{\mathrm{ef} 1} P_{1}}\right) \equiv-c_{3} \tau-c_{4} n \tau,
$$

where $c_{3}$ and $c_{4}$ are positive constants, and subscript $\mathrm{f}$ refers to the fuel gas $\left(\mathrm{H}_{2}\right.$ or $\left.\mathrm{CO}\right)$ in the binary gas system. The corresponding equation for the exhaust gas, after defining $\left(m_{\mathrm{e}} / m_{\mathrm{f}}\right)^{1 / 2} \equiv 2 h+1$, is

$$
\frac{\partial n_{\mathrm{e}}}{\partial x}=(2 h+1) c_{3} \tau+c_{4} n \tau \text {. }
$$

Adding Eqs. (4) and (5) yields the equation for $n$ and its solution,

$$
\frac{\partial n}{\partial x}=2 h c_{3} \tau, \quad n=n_{1}+2 h c_{3} \tau x .
$$

From the ideal gas law, $P=n k T$, we see that the total pressure $P$ increases linearly with $x$, in going through the anode from the plenum to the solid electrolyte.

Now that $n(x)$ is known, we can solve Eq. (4) for $n_{\mathrm{f}}$.

$$
\frac{\partial n_{\mathrm{f}}}{\partial x}=-c_{3} \tau-c_{4}\left(n_{1}+2 h c_{3} \tau x\right) \tau, \quad n_{\mathrm{f}}=n_{\mathrm{fp}}-\left(c_{3}+c_{4} n_{1}\right) \tau x-h c_{3} c_{4} \tau^{2} x^{2},
$$

where $n_{\mathrm{fp}}$ is the concentration of fuel gas in the plenum, which will be found below. We see that $n_{\mathrm{f}}$, and thus $n_{\mathrm{e}}=n-n_{\mathrm{f}}$, vary both linearly and quadratically with $x$.

If the cell current density is limited by anode saturation, designated as $i_{\text {as }}$ by $\mathrm{JV}$, then $i$ becomes $i_{\text {as }}$ in Eq. (4) and in the constants $c_{3}$ and $c_{4}$, which are designated $c_{3}$ as and $c_{4 \text { as }}$ for the anode saturation condition. In previous works [1,5] the approximation was made that in this limit the fuel concentration $n_{\mathrm{f}}$ reaches zero at the anode-electrolyte interface where $x=\tau w$. This approximation obviously cannot be exactly correct, because $i_{\text {as }}$ cannot be positive for zero fuel concentration, whereas the measured $i_{\text {as }}$ is positive.

To avoid making this unphysical approximation, we use results from our recent analysis of SOFC voltage $V$ as a function of current density $i[8]$. This relation is

$$
V(i)=\frac{U}{q}-\frac{k T}{q} \ln \left[\frac{(b+i)(d+i)^{1 / 2}}{(a-i)(c-i)^{1 / 2}}\right]-V_{\mathrm{ohm}} .
$$

Here, $U$ is the enthalpy for the gas-state reaction $\mathrm{H}_{2}+1 / 2 \mathrm{O}_{2} \rightarrow \mathrm{H}_{2} \mathrm{O}$ or $\mathrm{CO}+1 / 2 \mathrm{O}_{2} \rightarrow \mathrm{CO}_{2}$ at $800^{\circ} \mathrm{C}$ and 1 atm for all constituents, $q$ is the reaction charge transfer of $2 \mathrm{e}, k$ is Boltzmann's constant, $T$ is absolute temperature, and $V_{\text {ohm }}$ is the ohmic polarization. Unlike other $V(i)$ expressions in the literature (see [8]), Eq. (8) puts all four reaction attempt current densities $a, b, c, d$ on the same footing. The forward and reverse anode attempt current densities are $a$ and $b$, while the forward and reverse cathode attempt current densities are $c$ and $d$. These reaction attempt current densities are multiplied by reaction success probabilities which are functions of $k, T, q, i$, and anode and cathode contributions to $U$, to find anode and cathode contributions to $V$ in Eq. (8). Eq. (15) shows the form of $a$, and the other incoming gas reaction attempt current densities $b$ and $c$ have similar forms. Other authors (see [8]) have factors in the ln argument corresponding more or less to ours that involve $a, b$, and $c$, though these factors involve partial pressures whereas ours involve concentrations and molecular velocities. No other authors to our knowledge explicitly show a factor corresponding to our $(d+i)^{1 / 2}$ factor, where $d$ is the attempt current density corresponding to oxygen ions from the electrolyte combining to form $\mathrm{O}_{2}$ gas. This factor is much larger than the other three factors in the ln argument, and reduces the open-circuit emf considerably below the value of $1.214 \mathrm{~V}$ for $U / q$. Other authors instead implicitly incorporate the $-(k T / q) \ln \left[(d+i)^{1 / 2}\right]$ term as an entropy term as part of the reaction Gibbs energy $G$, and present the known $G$ in $G / q$ in place of our $U / q$ as the first term in $V(i)$. Fortunately, our $d$ expression [8] based on a molecular model for that reaction attempt rate gives approximately the correct open-circuit emf and thus provides a check on that reaction model.

The terminal voltage $V$ is often expressed as a sum of terms

$$
V=V_{0}-V_{\text {act }}-V_{\text {conc }}-V_{\text {ohm }}
$$


and each term can be found separately as shown below. The open-circuit emf $V_{0}$, from Eq. (8) with $i=V_{\mathrm{ohm}}=0$, and with $a, b, c, d$ set at their open-circuit values $a_{0}, b_{0}, c_{0}, d_{0}$, is

$$
V_{0}=\frac{U}{q}-\frac{k T}{q} \ln \frac{b_{0} d_{0}^{1 / 2}}{a_{0} c_{0}^{1 / 2}} .
$$

The activation polarization from Eq. (9) is given by $V_{\mathrm{act}}=V_{0}-V-V_{\mathrm{conc}}-V_{\mathrm{ohm}}$. It signifies the voltage drop required to provide a given $i$ for fixed gas concentrations at the electrode interfaces with the electrolyte. If we set $a=a_{0}$, etc. in Eq. (8) we eliminate $V_{\text {conc }}$ because the gas concentrations at the electrode interfaces with the electrolyte depend only on $a, b$, and $c$. If we also eliminate $V_{\text {ohm }}$ in Eq. (8), then Eq. (8) becomes

$$
V_{0}-V_{\mathrm{act}}=\frac{U}{q}-\frac{k T}{q} \ln \frac{\left(b_{0}+i\right)\left(d_{0}+i\right)^{1 / 2}}{\left(a_{0}-i\right)\left(c_{0}-i\right)^{1 / 2}},
$$

so $V_{\text {act }}$ is found by subtracting the Eq. (11) from the Eq. (10) expression,

$$
V_{\text {act }}=\frac{k T}{q} \ln \frac{a_{0} c_{0}^{1 / 2}\left(b_{0}+i\right)\left(d_{0}+i\right)^{1 / 2}}{b_{0} d_{0}^{1 / 2}\left(a_{0}-i\right)\left(c_{0}-i\right)^{1 / 2}} .
$$

Inserting expressions from Eqs. (8) and (12) into Eq. (9), we obtain

$$
V_{\text {conc }}=\frac{k T}{q} \ln \frac{\left(a_{0}-i\right)(b+i)\left(c_{0}-i\right)^{1 / 2}(d+i)^{1 / 2}}{(a-i)\left(b_{0}+i\right)(c-i)^{1 / 2}\left(d_{0}+i\right)^{1 / 2}} .
$$

The meaning of $V_{\text {conc }}$ is the additional voltage drop required because increasing $i$ causes changes in gas concentrations at the electrode interfaces with the electrolyte. An important fact, generally not mentioned, is that there are two contributions to $V_{\text {conc }}$. The one that usually comes to mind is the change with $i$ of gas concentrations as one goes across the electrodes. The other contribution is the dependence on $i$ of gas partial pressures and concentrations in the plenums which occurs even if there is no concentration change across the electrodes and the feed gas flow rates remain fixed. Both contributions are discussed below. Both $V_{\text {act }}$ and $V_{\text {conc }}$ take on the sign of $i$. The sum of $V_{\text {act }}$ and $V_{\text {conc }}$ is

$$
V_{\text {act }}+V_{\text {conc }}=\frac{k T}{q} \ln \frac{a_{0} c_{0}^{1 / 2}(b+i)(d+i)^{1 / 2}}{b_{0} d_{0}^{1 / 2}(a-i)(c-i)^{1 / 2}} .
$$

The saturation current density $i_{\text {as }}$ is defined as the current density at which $V$ drops to zero, but from both experiment and our $V(i)$ model [8] the voltage drops extremely rapidly toward $-\infty$ as $i$ goes through $i_{\text {as }}$. Accordingly, to a very good approximation, $i_{\text {as }}$ is the current density at which (an applied) $V$ approaches $-\infty$. From Eq. (8), this occurs for $i_{\text {as }}=a$.

Our model [8] gives a relation between $a$ and $n_{\mathrm{f}}$, allowing us to find the $n_{\mathrm{f}}$ value $n_{\text {fas }}$ attained at the anode saturation condition where $a=i_{\text {as }}$. This relation is

$$
a=\frac{1}{2} n_{\mathrm{f}}\left(\frac{k T}{m_{\mathrm{f}}}\right)^{1 / 2} q\left(1-v_{\mathrm{a}}\right) f_{\mathrm{a}},
$$

where $m_{\mathrm{f}}$ is the fuel molecule mass, $\left(k T / m_{\mathrm{f}}\right)^{1 / 2}$ is the fuel molecule average velocity component (through the anode pore) directed toward the triple phase boundary (TPB), $1-v_{\mathrm{a}}$ is the probability that an $\mathrm{O}^{2-}$ site at the anode TPB will be occupied (not vacant) so the fuel oxidation reaction can occur, and $f_{\mathrm{a}}$ is the probability that the fuel molecule strikes the electrolyte at an anode TPB. The Eq. (15) relation is model-dependent, but it seems that in any model there will be a linear or nearly linear relation between $a$ and $n_{\mathrm{f}}$.

Going back now to Eq. (7), we can no longer set $n_{\mathrm{f}}(x=L=\tau w)=0$, but instead we designate it as $n_{\mathrm{fas}}$. We then obtain a quadratic equation for $\tau^{2}$, valid for the anode saturation condition

$$
h c_{3 \mathrm{as}} c_{4 \mathrm{as}} w^{2} \tau^{4}+\left(c_{3 \mathrm{as}}+c_{4 \mathrm{as}} n_{1}\right) w \tau^{2}-n_{\mathrm{fpas}}+n_{\mathrm{fas}} \equiv A \tau^{4}+B \tau^{2}+C=0 .
$$

All constants in Eq. (16) except $C$ are positive, so in the solution

$$
\tau^{2}=\frac{-B \pm\left(B^{2}-4 A C\right)^{1 / 2}}{2 A},
$$

we must choose the + sign so that $\tau^{2}$ will be positive.

All constants in Eq. (16) have been defined except for the boundary conditions $n_{1}$ and $n_{\text {fpas }}$ for the total and fuel gas concentrations, respectively in the plenum at the outer anode surface, and $n_{\text {fas }}$ for the fuel gas concentration at the anode-electrolyte interface. For the JV experiments, $n_{1}=P_{1} / k T$ where $P_{1}=1 \mathrm{~atm}=1.015 \times 10^{5} \mathrm{~N} \mathrm{~m}^{-2}$ and $T=1073 \mathrm{~K}$, so $n_{1}=6.855 \times 10^{18} \mathrm{~cm}^{-3}$. 
To find $n_{\text {fpas }}$ we consider the total flow in and out of the anode plenum. From the discussion in JV p. A943 it appears that for all the binary and ternary systems displayed in JV Table 2, the metered gas inflow rate of $140 \mathrm{~mL} \mathrm{~min}^{-1}$ is for two gases, so that in the ternary systems the third gas is $\mathrm{H}_{2} \mathrm{O}$ generated at the anode-electrolyte interface. This total metered inflow rate of $140 \mathrm{~mL} \mathrm{~min}^{-1}$ at a temperature of $T_{\mathrm{in}}=273 \mathrm{~K}$ and at 1 sea level atmosphere is, from the ideal gas law,

$$
P_{1} \frac{\mathrm{d} V_{\text {in }}}{\mathrm{d} t}=k T_{\text {in }} \frac{\mathrm{d} N_{\text {in }}}{\mathrm{d} t}, \quad \text { so } \frac{\mathrm{d} N_{\text {in }}}{\mathrm{d} t} \equiv j_{1}=6.286 \times 10^{19} \text { molec s}^{-1} .
$$

$\mathrm{JV}$ did not specify the temperature at the metered inflow, but $T_{\mathrm{in}}=273 \mathrm{~K}$ fits their results best, based on solving for $m_{\mathrm{T}}$ from

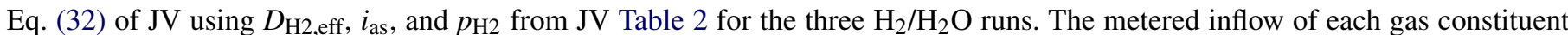
is found by multiplying the total metered inflow rate by the partial pressure fraction given or implied in JV Table 2. For instance, the first entry is $p_{\mathrm{f}}=0.2$ for $\mathrm{H}_{2}$, so $p_{\mathrm{e}}=0.8$ for $\mathrm{H}_{2} \mathrm{O}$. Though these Table 2 entries are in atmospheres, in this analysis we define these $p$ s as dimensionless partial pressure fractions. Then the respective metered inflow rates are: $j_{\mathrm{fm}}=p_{\mathrm{f}} j_{1}=1.257 \times 10^{19} \mathrm{~s}^{-1}$ and $j_{\mathrm{em}}=p_{\mathrm{e}} j_{1}=5.029 \times 10^{19} \mathrm{~s}^{-1}$. We assume here that the $p$ values in JV Table 2 are really $p^{\mathrm{o}}$ values, where $p^{\mathrm{o}}$ is the partial pressure of the metered incoming fuel or diluent as defined below Eq. (26) of JV.

To find the net inflow of each gas into the plenum, we must also consider the fuel gas outflow into the anode, and the exhaust gas inflow from the anode. These flows are equal and opposite, and are given by $j_{\mathrm{e}}=-j_{\mathrm{f}}=i S / q$, where $S$ is the active area of $1.1 \mathrm{~cm}^{2}$ and $q=3.2 \times 10^{-19} \mathrm{C}$. Using again the first entry in JV Table $2, i_{\text {as }}=1.3 \mathrm{~A} \mathrm{~cm}^{-2}$, we find $\mathrm{H}_{2}$ outflow $j_{\text {fas }}=-4.469 \times 10^{18} \mathrm{molec} \mathrm{s}^{-1}$ and $\mathrm{H}_{2} \mathrm{O}$ inflow jeas $=+4.469 \times 10^{18}$ molec s${ }^{-1}$. Comparison of the $\mathrm{H}_{2}$ outflow with the metered $\mathrm{H}_{2}$ inflow gives a fuel utilization $-j_{\text {fas }} / j_{\mathrm{fm}}$ of $35.5 \%$ for this example.

From the numbers in the previous two paragraphs, the net $\mathrm{H}_{2}$ inflow into the plenum is $j_{\text {fnet }}=j_{\mathrm{fm}}+j_{\text {fas }}=8.104 \times 10^{18} \mathrm{molec} \mathrm{s}^{-1}$ and the net $\mathrm{H}_{2} \mathrm{O}$ inflow is $j_{\text {enet }}=j_{\text {em }}+j_{\text {eas }}=5.476 \times 10^{19} \mathrm{molec} \mathrm{s}^{-1}$, for a total net inflow to the plenum of $j_{1}=6.286 \times 10^{19} \mathrm{~s}^{-1}$. The outflow from the plenum to the atmosphere must contain the corresponding fractions, $-j_{\text {fnet }} / j_{1}=0.1289$ for $\mathrm{H}_{2}$ and $j_{\text {enet }} / j_{1}=0.8711$ for $\mathrm{H}_{2} \mathrm{O}$, on a molecular basis. These values are considerably different from the corresponding metered inflow fractions, $p_{\mathrm{f}}=0.2$ and $p_{\mathrm{e}}=0.8$. To summarize the analytic expression for $j_{\text {fnet }}$ and $j_{\text {enet }}$,

$$
j_{\text {fnet }}=p_{\mathrm{f}} j_{1}-\frac{i S}{q} ; \quad j_{\text {enet }}=\left(1-p_{\mathrm{f}}\right) j_{1}+\frac{i S}{q} .
$$

One must consider whether the mole fractions in the plenum are the same as the mole fractions in the outflow. For openings small compared to the mean free path, this is not the case, because the outflow rate per molecule in the plenum is inversely proportional to the square root of the molecular mass [6]. However, it seems probable that the outflow arrangement provided by JV result in the mole fractions in the plenum being essentially the same as in the outflow. We assume that the gases in the plenum are well mixed and have the same mole fractions everywhere. Accordingly, in the plenum the fuel and exhaust concentrations under anode saturation conditions are

$$
n_{\mathrm{fpas}}=\left(\frac{j_{\mathrm{fas}}}{j_{1}}\right) n_{1}=\left(p_{\mathrm{f}}-\frac{i_{\mathrm{as}}}{i_{1}}\right) n_{1} ; \quad j_{\mathrm{epas}}=\left(\frac{j_{\mathrm{eas}}}{j_{1}}\right) n_{1}=\left(1-p_{\mathrm{f}}+\frac{i_{\mathrm{as}}}{i_{1}}\right) n_{1},
$$

where $p_{\mathrm{f}}$ and $i_{\mathrm{as}}$ are values taken from JV Table 2, and $i_{1}=j_{1} q / S=18.29 \mathrm{~A} \mathrm{~cm}^{-2}$.

The remaining parameter to determine is $n_{\text {fas }}$, the fuel gas concentration at the anode-electrolyte interface under the anode saturation condition. From Eq. (15), $n_{\mathrm{f}}$ remains proportional to $a$ as $i$ changes, because we assume the other parameters are independent of $i$. For open-circuit $(i=0)$ conditions, $n_{\mathrm{f}}$ at the interface equals $n_{\mathrm{f} 0}$ at the plenum, which from Eq. (20) will be $p_{\mathrm{f}} n_{1}$ for opencircuit. This known value for open-circuit $n_{\mathrm{f}}$ inserted into Eq. (15) provides a known value $a_{0}=p_{\mathrm{f}} a_{01}$ for the open-circuit value of $a$, where $a_{01}$ is the open-circuit value for $p_{\mathrm{f}}=1$. For the anode saturation condition, $a=i_{\text {as }}$, and because $n_{\mathrm{f}}$ is proportional to $a, n_{\mathrm{fas}}=n_{\mathrm{f} 0} a_{\mathrm{as}} / a_{0}=p_{\mathrm{f}} n_{1} i_{\mathrm{as}} / p_{\mathrm{f}} a_{01}=n_{1} i_{\mathrm{as}} / a_{01}$. Inserting this $n_{\mathrm{fas}}$ value and the above $n_{\text {fpas }}$ value into Eq. (16) yields the following equation for $\tau^{2}$ in terms of known parameters

$$
h c_{3 \mathrm{as}} c_{4 \mathrm{as}} w^{2} \tau^{4}+\left(c_{3 \mathrm{as}}+c_{4 \mathrm{as}} n_{1}\right) w \tau^{2}-\left(p_{\mathrm{f}}-\frac{i_{\text {as }}}{i_{1}}\right) n_{1}+n_{1} \frac{i_{\text {as }}}{a_{01}} \equiv A \tau^{4}+B \tau^{2}+C=0 .
$$

In our previous analysis [5] the $n_{1} i_{\text {as }} / a_{01}$ term was missing.

\section{Results for binary gas input}

We are now in a position to use Eqs. (21) and (17) to find $\tau$

$$
\tau=\left\{\frac{-B+\left(B^{2}-4 A C\right)^{1 / 2}}{2 A}\right\}^{1 / 2} .
$$



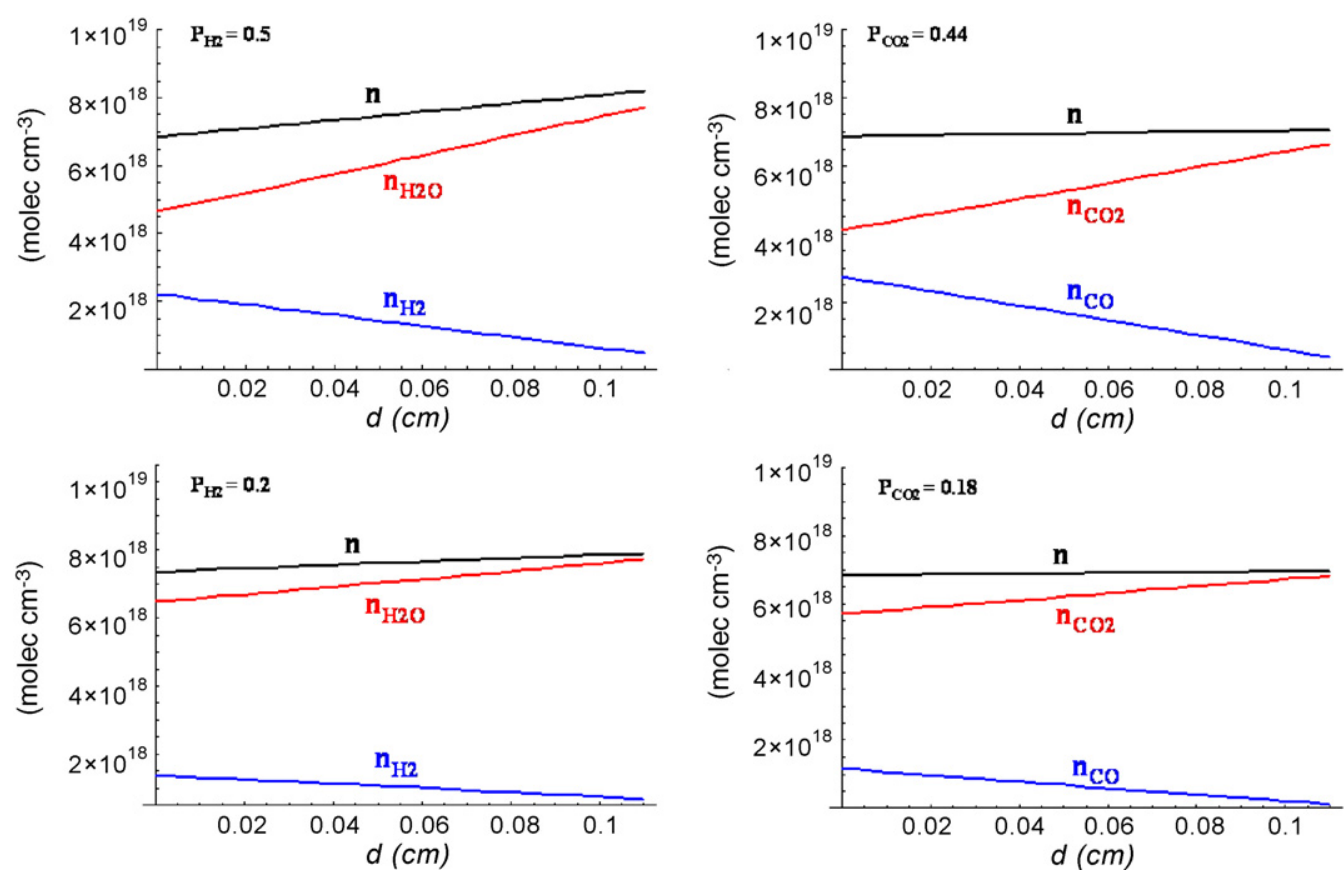

Fig. 2. Plots of gas concentrations vs. position in anode for some binary gas inputs under anode limiting current conditions.

We begin by summarizing the constants that appear directly or indirectly in Eq. (16), and that do not change from run to run. They are provided here to aid the reader who may want to check the numerical results of this study that appear in Table 1 and Fig. 2. The meanings of the symbols are explained above where the symbols first appear.

$$
\begin{aligned}
& \phi=0.54, \quad q=3.2 \times 10^{-19} \mathrm{C}, \quad k=1.38 \times 10^{-23} \mathrm{~J} \mathrm{~K}^{-1}, \quad T=1073 \mathrm{~K}, \quad P_{1}=1.015 \times 10^{5} \mathrm{~N} \mathrm{~m}^{-2}, \\
& w=0.11 \mathrm{~cm}, \quad n_{1}=6.855 \times 10^{18} \mathrm{~cm}^{-3}, \quad i_{1}=18.29 \mathrm{Acm}^{-2}, \quad a_{01}=48.4 \mathrm{Acm}^{-2}, \\
& D_{\mathrm{Kf}}=11.3 \mathrm{~cm}^{2} \mathrm{~s}^{-1}\left(\mathrm{H}_{2}\right) ; 3.00 \mathrm{~cm}^{2} \mathrm{~s}^{-1}(\mathrm{CO}), \\
& D_{\mathrm{ef} 1}=7.704 \mathrm{~cm}^{2} \mathrm{~s}^{-1}\left(\mathrm{H}_{2}, \mathrm{H}_{2} \mathrm{O}\right)=1.408 \mathrm{~cm}^{2} \mathrm{~s}^{-1}\left(\mathrm{CO}, \mathrm{CO}_{2}\right), \\
& \frac{c_{3 \mathrm{as}}}{i_{\mathrm{as}}}=\frac{1}{\phi q D_{\mathrm{Kf}}}=5.121 \times 10^{17} \mathrm{~s} \mathrm{C}^{-1} \mathrm{~cm}^{-2}\left(\mathrm{H}_{2}\right)=1.929 \times 10^{18} \mathrm{~s} \mathrm{C}^{-1} \mathrm{~cm}^{-2}(\mathrm{CO}), \\
& \frac{c_{4 \mathrm{as}}}{i_{\mathrm{as}}}=\frac{k T}{\phi q D_{\mathrm{ef} 1} P_{1}}=0.1096 \mathrm{~cm} \mathrm{~s}^{-1}\left(\mathrm{H}_{2}\right)=0.5994 \mathrm{~cm} \mathrm{~s}^{-1}(\mathrm{CO}), \\
& h=\frac{\left(m_{\mathrm{e}} / m_{\mathrm{f}}\right)^{1 / 2}-1}{2}=1\left(\mathrm{H}_{2}\right),=0.1268(\mathrm{CO}), \\
& n_{\mathrm{fpas}}=-C=\left(p_{\mathrm{f}}-\frac{i_{\mathrm{as}}}{i_{1}}-\frac{i_{\mathrm{as}}}{a_{01}}\right) n_{1}, \quad A=h c_{3 \mathrm{as}} c_{4 \mathrm{as}} w^{2}, \quad B=\left(c_{3 \mathrm{as}}+c_{4 \mathrm{as}} n_{1}\right) w .
\end{aligned}
$$

We carry out a sample calculation for the first entry in JV Table 2, for the $\mathrm{H}_{2} / \mathrm{H}_{2} \mathrm{O}$ binary system, for which $p_{\mathrm{f}}=0.2$ and $i_{\mathrm{as}}=1.3 \mathrm{~A} \mathrm{~cm}^{-2}$. Various parameters used in this calculation and whose meanings are explained above are

$$
\begin{aligned}
& a_{01}=48.4 \mathrm{Acm}^{-2}, \quad a_{0}=p_{\mathrm{f}} a_{01}=9.68 \mathrm{Acm}^{-2} \quad \text { from our } V(i) \text { analysis [8] } \\
& c_{3 \mathrm{as}}=6.657 \times 10^{17} \mathrm{~cm}^{-4}, \quad c_{4 \mathrm{as}}=0.1425 \mathrm{~cm}^{-1}, \quad A=1.148 \times 10^{15} \mathrm{~cm}^{-3}, \quad B=1.807 \times 10^{17} \mathrm{~cm}^{-3}, \\
& C=-6.996 \times 10^{17} \mathrm{~cm}^{-3}\left(\text { Ref. [5] value }-8.836 \times 10^{17} \mathrm{~cm}^{-3}\right), \\
& \left.\left(B^{2}-4 A C\right)^{1 / 2}=1.894 \times 10^{17} \mathrm{~cm}^{-3} \text { (Ref. [5] value } 1.916 \times 10^{17} \mathrm{~cm}^{-3}\right), \\
& \tau=1.944 \text { (Ref. [5] value 2.179), JV } \tau^{1 / 2}=2.211, \quad \mathrm{JV} \tau=4.89 .
\end{aligned}
$$

These results and those for the other two $\mathrm{H}_{2} / \mathrm{H}_{2} \mathrm{O}$ data sets from JV appear in Table 1.

It is highly encouraging that our $\tau$ values agree well with each other, and especially that their small magnitudes seem very reasonable for the high-porosity $(\phi=0.54)$ anode that JV investigated. They agree fairly well also with the JV results in the $\left(\tau_{\mathrm{JV}}\right)^{1 / 2}$ column, where it is taken into account that what JV call tortuosity in the $\tau_{\mathrm{JV}}$ column is the tortuosity factor [2] whose square root is what we and others call tortuosity. To calculate the $\tau$ values for the four data sets from JV for the $\mathrm{CO} / \mathrm{CO}_{2}$ binary system, the above 
analysis is unchanged except that subscripts $\mathrm{f}$ and e refer to $\mathrm{CO}$ and $\mathrm{CO}_{2}$, respectively. The tortuosity results are given in Table 1 below.

We see in Table 1 that our previous [5] analysis results $\left[\tau\left(n_{\mathrm{fas}}=0\right)\right]$ which range from about 2.1 to 2.9 agree better than our present results $[\tau]$ which range from about 1.9 to 2.7 , with the Jiang-Virkar [1] results $\left[\left(\tau_{\mathrm{JV}}\right)^{1 / 2}\right]$ which range from about 2.2 to 2.9. This occurs because our previous analysis differed from theirs only by our allowing total pressure to vary along the pore, and by consequences of that variation. Our present analysis also takes into account that the fuel concentration is nonzero under anode saturation conditions, unlike our previous analysis and that of Jiang and Virkar. The result as seen in Table 1 is that our present tortuosity values are smaller than our previous values by roughly $10 \%$. We consider that these lower values are more accurate, but it must be remembered that the previous analyses by JV and ourselves were independent of a model for $V(i)$. Our confidence on the approximate validity of this $10 \%$ reduction is based on the accuracy with which our $V(i)$ model [8] fits the $V(i)$ curves of JV for the $\mathrm{H}_{2} / \mathrm{H}_{2} \mathrm{O}$ binary system.

Plots of gas concentrations vs. position in the anode appear in Fig. 2 for the lowest and highest $\mathrm{H}_{2}$ and $\mathrm{CO}$ fuel concentrations in the anode plenum. The total concentration, and thus total pressure, has much stronger position dependence for $\mathrm{H}_{2}$ than for $\mathrm{CO}$ fuel. This strong dependence of total pressure on position in the anode shows that the commonly made assumption of uniform total pressure across the anode is a serious approximation. The quadratic contribution to the position dependences for the various fuel and exhaust concentrations is barely visible.

\section{Calculation for ternary gas input}

The ternary systems consisted of incoming $\mathrm{H}_{2}$ fuel diluted with $\mathrm{He}, \mathrm{N}_{2}$, or $\mathrm{CO}_{2}$. They are ternary because $\mathrm{H}_{2} \mathrm{O}$ (steam) "exhaust" is produced at the anode-electrolyte interface. We will compare the JV calculations of tortuosity $\tau$ with values found from our analysis.

In the preceding analysis of the binary systems, we used subscripts e and f for exhaust and fuel gases, respectively. For the ternary systems, we will use subscripts $\mathrm{d}$ for the $\mathrm{He}, \mathrm{N}_{2}$, or $\mathrm{CO}_{2}$ diluent gases, e for the $\mathrm{H}_{2} \mathrm{O}$ exhaust gas, and $\mathrm{f}$ for the $\mathrm{H}_{2}$ fuel gas.

We note that for $\mathrm{H}_{2}$ fuel and $\mathrm{H}_{2} \mathrm{O}$ exhaust, in the Stefan-Maxwell equations the molar flow rates obey $N_{\mathrm{d}}=0$ and $N_{\mathrm{e}}=-N_{\mathrm{f}}$ and we denote $N_{\mathrm{f}}$ by $N$. Then Eq. (1) becomes

$$
\begin{aligned}
& \frac{N}{D_{\mathrm{Kf}}}+\frac{X_{\mathrm{d}} N}{D_{\mathrm{df}}}+\frac{\left(X_{\mathrm{f}}+X_{\mathrm{e}}\right) N}{D_{\mathrm{ef}}}=-\frac{1}{R T} \frac{\mathrm{d}\left(P X_{\mathrm{f}}\right)}{\mathrm{d} x}, \\
& -\frac{N}{D_{\mathrm{Ke}}}-\frac{X_{\mathrm{d}} N}{D_{\mathrm{de}}}-\frac{\left(X_{\mathrm{f}}+X_{\mathrm{e}}\right) N}{D_{\mathrm{ef}}}=-\frac{1}{R T} \frac{\mathrm{d}\left(P X_{\mathrm{e}}\right)}{\mathrm{d} x}, \\
& -\frac{X_{\mathrm{d}} N}{D_{\mathrm{df}}}+\frac{X_{\mathrm{d}} N}{D_{\mathrm{de}}}=-\frac{1}{R T} \frac{\mathrm{d}\left(P X_{\mathrm{d}}\right)}{\mathrm{d} x} .
\end{aligned}
$$

The third equation, for the diluent gas, is absent for binary systems.

First we convert to molecular units by multiplying through by Avogadro's number. This converts $N$ to $J$, which has units of molecules $\mathrm{cm}^{-2} \mathrm{~s}^{-1}$, and converts $R$ to $k$ which is Boltzmann's constant. Thus $1 / R T$ becomes $1 / k T$. From the ideal gas law, $P=n k T$, so $(1 / k T) \mathrm{d}\left(P X_{\mathrm{f}}\right) / \mathrm{d} x=\mathrm{d}\left(n X_{\mathrm{f}}\right) / \mathrm{d} x=\mathrm{d} n_{\mathrm{f}} / \mathrm{d} x$, etc. These are the concentration gradients in $\mathrm{cm}^{-4}$. Here $n$ is the total gas concentration and $n_{\mathrm{f}}$, etc. are the partial concentrations, in $\mathrm{cm}^{-3}$.

We can then write Eq. (25) in terms of molecular units as

$$
\begin{aligned}
\frac{\mathrm{d} n_{\mathrm{f}}}{\mathrm{d} x} & =-\frac{J}{D_{\mathrm{Kf}}}-\frac{J X_{\mathrm{d}}}{D_{\mathrm{df}}}-\frac{J\left(X_{\mathrm{e}}+X_{\mathrm{f}}\right)}{D_{\mathrm{ef}}}, \\
\frac{\mathrm{d} n_{\mathrm{e}}}{\mathrm{d} x} & =\frac{J}{D_{\mathrm{Ke}}}+\frac{J X_{\mathrm{d}}}{D_{\mathrm{de}}}+\frac{J\left(X_{\mathrm{e}}+X_{\mathrm{f}}\right)}{D_{\mathrm{ef}}}, \\
\frac{\mathrm{d} n_{\mathrm{d}}}{\mathrm{d} x} & =\frac{J X_{\mathrm{d}}}{D_{\mathrm{df}}}-\frac{J X_{\mathrm{d}}}{D_{\mathrm{de}}}
\end{aligned}
$$

The quoted $D_{i j}$ values in JV Table 1 , which we call $D_{i j 1}$, are based on 1 atmosphere pressure, which we label $P_{1}$. Accordingly, using the ideal gas law $P=n k T$, noting that $P$ is in the denominator of $D_{i j}$, and letting $n_{1}$ be the total gas concentration at $T=1073 \mathrm{~K}$,

$$
\frac{1}{D_{i j}}=\frac{P}{P_{1} D_{i j 1}}=\frac{n}{n_{1} D_{i j 1}} .
$$


Finally, we note that $X_{\mathrm{i}}=n_{\mathrm{i}} / n$, etc., so we can rewrite Eq. (26) as

$$
\begin{aligned}
\frac{\mathrm{d} n_{\mathrm{f}}}{\mathrm{d} x} & =-\frac{J}{D_{\mathrm{Kf}}}-\frac{J}{n_{1}}\left[\frac{n_{\mathrm{d}}}{D_{\mathrm{df} 1}}+\frac{\left(n_{e}+n_{f}\right)}{D_{\mathrm{ef} 1}}\right], \\
\frac{\mathrm{d} n_{\mathrm{e}}}{\mathrm{d} x} & =\frac{J}{D_{\mathrm{Ke}}}+\frac{J}{n_{1}}\left[\frac{n_{\mathrm{d}}}{D_{\mathrm{de} 1}}+\frac{n_{\mathrm{e}}+n_{\mathrm{f}}}{D_{\mathrm{ef} 1}}\right], \\
\frac{\mathrm{d} n_{\mathrm{d}}}{\mathrm{d} x} & =-\left(\frac{J n_{\mathrm{d}}}{n_{1}}\right)\left(\frac{1}{D_{\mathrm{de} 1}}-\frac{1}{D_{\mathrm{df} 1}}\right) .
\end{aligned}
$$

Adding these three equations and noting that $n_{\mathrm{d}}+n_{\mathrm{e}}+n_{\mathrm{f}}=n$ yields an equation for $n$ and its solution,

$$
\frac{\mathrm{d} n}{\mathrm{~d} x}=J\left(\frac{1}{D_{\mathrm{Ke}}}-\frac{1}{D_{\mathrm{Kf}}}\right), \quad n=n_{1}\left(1+c_{5} x\right) ; \quad c_{5} \equiv\left(\frac{J}{n_{1}}\right)\left(\frac{1}{D_{\mathrm{Ke}}}-\frac{1}{D_{\mathrm{Kf}}}\right)>0 .
$$

In solving the $\mathrm{d} n_{\mathrm{d}} / \mathrm{d} x$ equation in Eq. (28) for $n_{\mathrm{d}}$, we get

$$
n_{\mathrm{d}}=n_{\mathrm{dp}} \exp \left(c_{7} x\right) ; \quad c_{7} \equiv-\left(\frac{J}{n_{1}}\right)\left(\frac{1}{D_{\mathrm{de} 1}}-\frac{1}{D_{\mathrm{df} 1}}\right)<0 .
$$

Jiang and Virkar [1] pointed out this exponential nature of the diluent gas concentration dependence on position, but omitted using this dependence to simplify their calculations. This non-vanishing concentration dependence on $x$ may seem surprising because the diluent gas has no net flow in the pores. It occurs because of the unlike masses of the fuel and exhaust molecules that the diluent molecules collide with. The $>0$ and $<0$ relations occur in Eqs. (29) and (30), respectively because for the systems studied, the exhaust molecule is more massive than the fuel molecule and the inverse Knudsen and binary diffusion coefficients increase with molecular mass. Accordingly, Eq. (29) tells us that total gas concentration increases linearly with $x$, whereas Eq. (30) tells us that diluent gas concentration decreases exponentially with $x$.

Because $n_{1}$ and $n_{\mathrm{dp}}$ are known as explained below, we can solve for $n_{\mathrm{e}}$ in terms of $n_{\mathrm{f}}$

$$
n_{\mathrm{e}}=n-n_{\mathrm{d}}-n_{\mathrm{f}}=n_{1}\left(1+c_{5} x\right)-n_{\mathrm{dp}} \exp \left(c_{7} x\right)-n_{\mathrm{f}} .
$$

Then the $\mathrm{d} n_{\mathrm{f}} / \mathrm{d} x$ equation in Eq. (28) becomes

$$
\frac{\mathrm{d} n_{\mathrm{f}}}{\mathrm{d} x}=-J\left[\frac{1}{D_{\mathrm{Kf}}}+\frac{1+c_{5} x}{D_{\mathrm{ef} 1}}\right]+\frac{J n_{\mathrm{dp}}}{n_{1}}\left(\frac{1}{D_{\mathrm{ef} 1}}-\frac{1}{D_{\mathrm{df} 1}}\right) \exp \left(c_{7} x\right)
$$

The solution to this equation can be written as

$$
n_{\mathrm{f}}=n_{\mathrm{fp}}+n_{\mathrm{dp}} g\left[1-\exp \left(c_{7} x\right)\right]-\left(\frac{J}{D_{\mathrm{Kf}}}\right) x-\frac{J}{D_{\mathrm{ef} 1}}\left(x+\frac{1}{2} c_{5} x^{2}\right) ; \quad g \equiv \frac{\left(1 / D_{\mathrm{ef} 1}\right)-\left(1 / D_{\mathrm{df} 1}\right)}{\left(1 / D_{\mathrm{de} 1}\right)-\left(1 / D_{\mathrm{df} 1}\right)} .
$$

Eq. (33) is especially useful under the condition of anode saturation. Under that condition we previously [5] set $n_{\mathrm{f}}$ equal to zero at the electrolyte interface, where $x=L=\tau w$, but as explained in the binary system analysis, we now set $n_{\mathrm{f}}=n_{\mathrm{fas}}$, its nonzero value for the current density value $i_{\text {as }}$ for which the output voltage $V$ drops rapidly through zero on its way to $-\infty$.

We also need expressions for $n_{\mathrm{dp}}$ and $n_{\mathrm{fp}}$ under the anode saturation condition. To find these, we note that under this condition the net flow rates in molec $\mathrm{s}^{-1}$ into the anode plenum are

$$
j_{\mathrm{d}}=j_{1} p_{\mathrm{d}}, \quad j_{\mathrm{e}}=\frac{i_{\mathrm{as}} S}{q}, \quad j_{\mathrm{f}}=j_{1}\left(1-p_{\mathrm{d}}\right)-\frac{i_{\mathrm{as}} S}{q} .
$$

The total of these flow rates into the plenum is simply $j_{1}$. Here, $i_{\text {as }}$ is the anode saturation current density as given in JV Table 2 , $S$ is the electrode area of $1.1 \mathrm{~cm}^{2}, q$ is the charge of $3.2 \times 10^{-19} \mathrm{C}$ per gas molecule created or destroyed at the anode/electrolyte interface, and $p_{\mathrm{d}}$ is the fractional partial pressure of the diluent gas, given in JV Table 2 as $p_{\mathrm{He}}, p_{\mathrm{N}_{2}}$, or $p_{\mathrm{CO}_{2}}$. Note that the superscript o used in Eq. (26) of JV for metered incoming gas is missing in JV Table 2. One finds the total incoming molecular flow rate $j_{1}$ from the ideal gas law, $P_{1} V_{\text {in }}=N_{\text {in }} k T_{\text {in }}$, using

$$
j_{1} \equiv \frac{\mathrm{d} N_{\mathrm{in}}}{\mathrm{d} t}=\frac{P_{1}}{k T_{\text {in }}} \frac{\mathrm{d} V_{\mathrm{in}}}{\mathrm{d} t}
$$

using $\mathrm{d} V_{\text {in }} / \mathrm{d} t$ as $140 \mathrm{~cm}^{3} \mathrm{~min}^{-1}$ as stated by JV for all experiments. In Eq. (35) we use $T_{\text {in }}=273 \mathrm{~K}$ to best fit the results of JV using their calculational method, so $j_{1}=6.286 \times 10^{19}$ molec s$^{-1}$. 
As discussed for the binary systems, the net flow rates into the plenum equal the flow rates out of the plenum exit orifice, and these exit orifice flow rates are proportional to the gas concentrations in the plenum, so

$$
n_{\mathrm{dp}}=\frac{j_{\mathrm{d}}}{j_{1}} n_{1}=p_{\mathrm{d}} n_{1}, \quad n_{\mathrm{fp}}=\frac{j_{\mathrm{f}}}{j_{1}} n_{1}=\left(1-p_{\mathrm{d}}-\frac{i_{\mathrm{as}} S}{q j_{1}}\right) n_{1} \equiv\left(1-p_{\mathrm{d}}-\frac{i_{\mathrm{as}}}{i_{1}}\right) n_{1} ; \quad i_{1} \equiv \frac{q j_{1}}{S} .
$$

Finally, we find $J$ under the anode saturation condition, as discussed for the binary systems

$$
J_{\mathrm{as}}=\frac{i_{\mathrm{as}} \tau}{\phi q} .
$$

We can now write Eq. (33) under the anode saturation condition and evaluated at $x=\tau w$. From Eqs. (29), (30), and (37), we see that $c_{5}$ and $c_{7}$ depend linearly on $\tau$. Accordingly, we must use the $\tau$-independent factors $c_{6}=c_{5} w / \tau$ and $c_{8}=c_{7} w / \tau$ to display the true $\tau$ dependence.

$$
n_{\mathrm{fas}}(x=L=\tau w)=\left(1-p_{\mathrm{d}}-\frac{i_{\mathrm{as}}}{i_{1}}\right) n_{1}+p_{\mathrm{d}} n_{1} g\left[1-\exp \left(c_{8} \tau^{2}\right)\right]-\frac{i_{\mathrm{as}} w}{\phi q D_{\mathrm{Kf}}} \tau^{2}-\frac{i_{\mathrm{as}} w}{\phi q D_{\mathrm{ef} 1}}\left(\tau^{2}+\frac{1}{2} c_{6} \tau^{4}\right) .
$$

This is a transcendental equation for $\tau^{2}$ that can be solved numerically. Written out explicitly by substituting for $c_{5}, c_{7}$, and $g$ from Eqs. (23), (24), and (27), respectively, it appears as

$$
\begin{aligned}
& \left(1-p_{\mathrm{d}}-\frac{i_{\mathrm{as}}}{i_{1}}-\frac{i_{\mathrm{as}}}{a_{01}}\right) n_{1}-\frac{i_{\mathrm{as}} w}{\phi q D_{\mathrm{Kf}}} \tau^{2}-\frac{i_{\mathrm{as}} w}{\phi q D_{\mathrm{ef} 1}}\left[\tau^{2}+\frac{1}{2}\left(\frac{i_{\mathrm{as}} w}{\phi q n_{1}}\right)\left(\frac{1}{D_{\mathrm{Ke}}}-\frac{1}{D_{\mathrm{Kf}}}\right) \tau^{4}\right] \\
& +p_{\mathrm{d}} n_{1}\left[\frac{\left(1 / D_{\mathrm{ef} 1}\right)-\left(1 / D_{\mathrm{df} 1}\right)}{\left(1 / D_{\mathrm{de} 1}\right)-\left(1 / D_{\mathrm{df} 1}\right)}\right]\left\{1-\exp \left[\frac{-i_{\mathrm{as}} w}{\phi q n_{1}}\left(\frac{1}{D_{\mathrm{de} 1}}-\frac{1}{D_{\mathrm{df} 1}}\right) \tau^{2}\right]\right\}=0 .
\end{aligned}
$$

In Eq. (39), we use $n_{\mathrm{fas}}=n_{1} i_{\mathrm{as}} / a_{01}$ as we did for the binary system.

\section{Results for ternary gas input}

The relations in the equations below are useful in solving Eq. (39) numerically for the tortuosity $\tau$ values presented in Table 2 and Fig. 3. The $D_{\mathrm{de} 1}$ values below are calculated from Eq. (2.22) and Tables 2.2 and 2.3 of Mass Transfer [7]. The constants that remain the same from one run to the next are
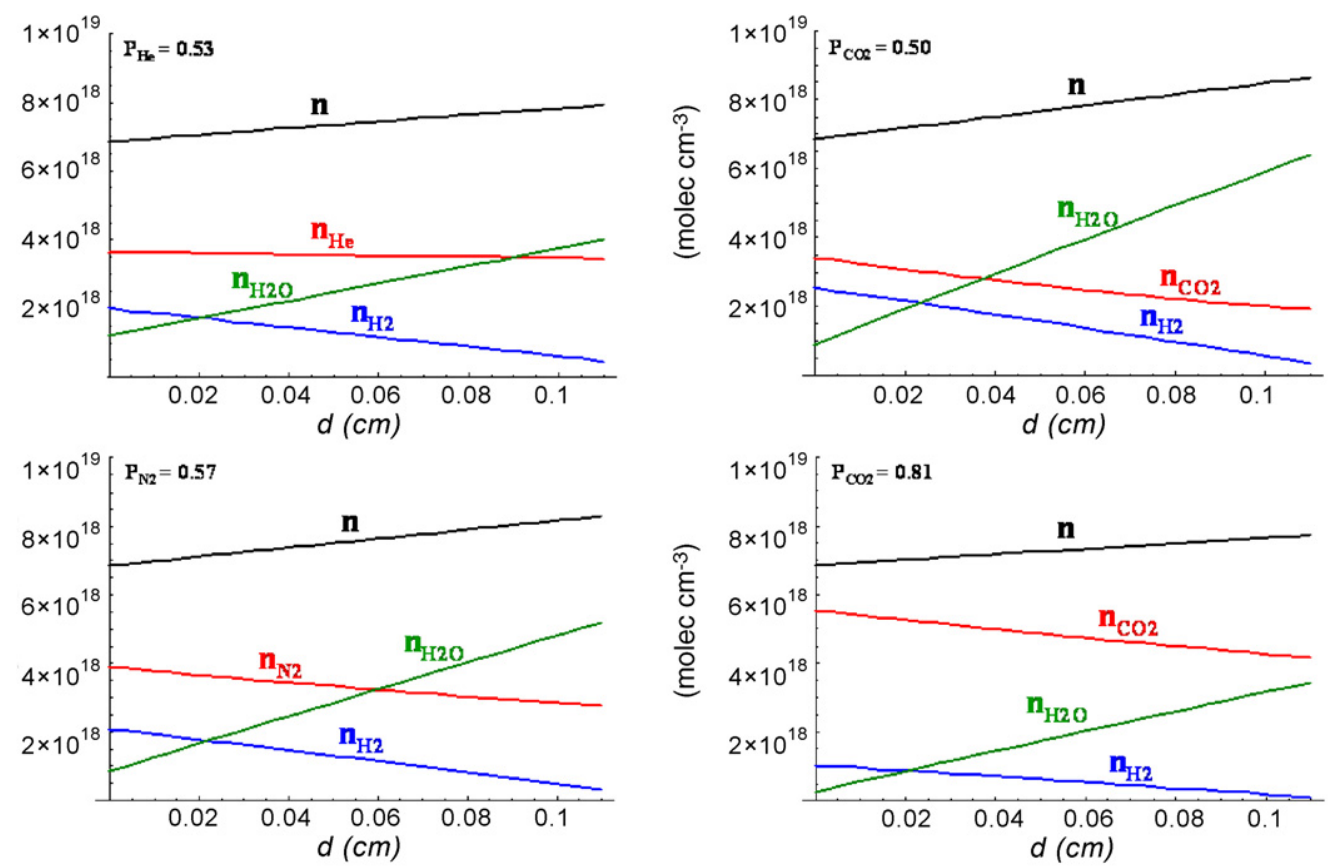

Fig. 3. Plots of gas concentrations $v s$. position in anode for some ternary gas inputs under anode limiting current conditions. 


$$
\begin{aligned}
& D_{\mathrm{Ke}}=3.767, \quad D_{\mathrm{Kf}}=11.3, \quad D_{\mathrm{ef} 1}=7.704 \mathrm{~cm}^{2} \mathrm{~s}^{-1} \text {, } \\
& D_{\text {de1 }}=7.462, \quad D_{\text {df1 }}=13.29 \text { for } \mathrm{d}=\mathrm{He} \text {, } \\
& D_{\text {de } 1}=2.21, \quad D_{\text {df } 1}=6.303 \text { for } \mathrm{d}=\mathrm{N}_{2} \text {, } \\
& D_{\text {de1 }}=1.73, \quad D_{\text {df1 }}=5.56 \text { for } \mathrm{d}=\mathrm{CO}_{2} \text {, } \\
& \frac{1}{D_{\mathrm{de} 1}}-\frac{1}{D_{\mathrm{df} 1}}=0.05877 \mathrm{~s} \mathrm{~cm}^{-2} \text { for } \mathrm{d}=\mathrm{He} \text {, } \\
& \frac{1}{D_{\mathrm{de} 1}}-\frac{1}{D_{\mathrm{df} 1}}=0.2938 \text { for } \mathrm{d}=\mathrm{N}_{2} \text {, } \\
& \frac{1}{D_{\mathrm{de} 1}}-\frac{1}{D_{\mathrm{df} 1}}=0.3982 \text { for } \mathrm{d}=\mathrm{CO}_{2} \text {, } \\
& \frac{1}{D}-\frac{1}{D_{\mathrm{df} 1}}=0.0546 \mathrm{~s} \mathrm{~cm}^{-2} \text { for } \mathrm{d}=\mathrm{He} \text {, } \\
& \frac{1}{D_{\text {ef } 1}}-\frac{1}{D_{\text {df1 }}}=-0.0289 \text { for } \mathrm{d}=\mathrm{N}_{2} \text {, } \\
& \frac{1}{D_{\text {ef } 1}}-\frac{1}{D_{\text {df } 1}}=-0.0501 \text { for } \mathrm{d}=\mathrm{CO}_{2} \text {, } \\
& \frac{1}{D_{\mathrm{Kf}}}+\frac{1}{D_{\mathrm{ef} 1}}=0.2183 \mathrm{~s} \mathrm{~cm}^{-2}, \quad \frac{1}{D_{\mathrm{ef} 1}}\left(\frac{1}{D_{\mathrm{Ke}}}-\frac{1}{D_{\mathrm{Kf}}}\right)=0.02297 \mathrm{~s}^{2} \mathrm{~cm}^{-4}, \\
& P_{1}=1.015 \times 10^{5} \mathrm{Nm}^{-2}, \quad k=1.38 \times 10^{-23} \mathrm{JK}, \quad T=1073 \mathrm{~K}, \quad n_{1}=\frac{P_{1}}{k T}=6.855 \times 10^{18} \mathrm{~cm}^{-3}, \\
& w=0.11 \mathrm{~cm}, \quad S=1.1 \mathrm{~cm}^{2}, \quad \phi=0.54, \quad q=3.2 \times 10^{-19} \mathrm{C}, \quad j_{1}=6.286 \times 10^{19} \mathrm{~s}^{-1}, \\
& a_{01}=48.4 \mathrm{~A} \mathrm{~cm}^{-2}, \quad i_{1}=\frac{q j_{1}}{S}=18.29 \mathrm{~A} \mathrm{~cm}^{-2}, \quad \frac{w}{\phi q}=6.366 \times 10^{17} \mathrm{~cm} \mathrm{C}^{-1}, \\
& \frac{(w / \phi q)^{2}}{2 n_{1}}=2.956 \times 10^{16} \mathrm{~cm}^{5} \mathrm{C}^{-2}, \quad \frac{w}{\phi q D_{\mathrm{Kf}}}=5.634 \times 10^{16}, \\
& \frac{w}{\phi q D_{\mathrm{ef} 1}}=8.263 \times 10^{16} \mathrm{~s} \mathrm{~cm}^{-1} \mathrm{C}^{-1}, \quad \frac{w}{2 \phi q n_{1}}\left(\frac{1}{D_{\mathrm{Ke}}}-\frac{1}{D_{\mathrm{Kf}}}\right)=0.008217 \mathrm{~cm}^{2} \mathrm{~s} \mathrm{C}^{-1}, \\
& \frac{w}{\phi q n_{1}}\left(\frac{1}{D_{\mathrm{de} 1}}-\frac{1}{D_{\mathrm{df} 1}}\right)=0.005458 \mathrm{~cm}^{2} \mathrm{~s} \mathrm{C}^{-1} \text { for } \mathrm{d}=\mathrm{He} \text {, } \\
& \frac{w}{\phi q n_{1}}\left(\frac{1}{D_{\mathrm{de} 1}}-\frac{1}{D_{\mathrm{df} 1}}\right)=0.02728 \mathrm{~cm}^{2} \mathrm{sC}^{-1} \text { for } \mathrm{d}=\mathrm{N}_{2} \text {, } \\
& \frac{w}{\phi q n_{1}}\left(\frac{1}{D_{\mathrm{de} 1}}-\frac{1}{D_{\mathrm{df1} 1}}\right)=0.03698 \mathrm{~cm}^{2} \mathrm{~s} \mathrm{C}^{-1} \text { for } \mathrm{d}=\mathrm{CO}_{2} \text {. }
\end{aligned}
$$

Though Eq. (38) is easy to solve exactly for $\tau$ by numerical methods, a sufficiently accurate analytical expression for $\tau$ can be obtained by approximating the exponential term by a quadratic expression,

$$
\exp \left(c_{8} \tau^{2}\right) \cong 1+c_{8} \tau^{2}+\frac{1}{2}\left(c_{8}\right)^{2} \tau^{4} .
$$

Then Eq. (38), or Eq. (39), becomes a quadratic equation in $\tau^{2}$ and can be solved for $\tau$ in the usual manner. This quadratic equation, its solution, and its $\mathrm{A}^{\prime}, \mathrm{B}^{\prime}$ and $\mathrm{C}^{\prime}$ coefficients are

$$
\begin{aligned}
& A^{\prime} \tau^{4}+B^{\prime} \tau^{2}+C^{\prime}=0, \quad \tau=\left\{\frac{-B^{\prime} \pm\left(B^{\prime 2}-4 A^{\prime} C^{\prime}\right)^{1 / 2}}{2 A^{\prime}}\right\}^{1 / 2}, \\
& A^{\prime}=-\left[\frac{\left(i_{\mathrm{as}} w / \phi q\right)^{2}}{2 n_{1}}\right]\left[\frac{1}{D_{\mathrm{ef} 1}}\left(\frac{1}{D_{\mathrm{Ke}}}-\frac{1}{D_{\mathrm{Kf}}}\right)+p_{\mathrm{d}}\left(\frac{1}{D_{\mathrm{de} 1}}-\frac{1}{D_{\mathrm{df} 1}}\right)\left(\frac{1}{D_{\mathrm{ef} 1}}-\frac{1}{D_{\mathrm{df} 1}}\right)\right], \\
& B^{\prime}=-\frac{i_{\mathrm{as}} w}{\phi q}\left[\frac{1}{D_{\mathrm{Kf}}}+\frac{1-p_{\mathrm{d}}}{D_{\mathrm{ef} 1}}+\frac{p_{\mathrm{d}}}{D_{\mathrm{df} 1}}\right], \quad C^{\prime}=\left(1-p_{\mathrm{d}}-\frac{i_{\mathrm{as}}}{i_{1}}-\frac{i_{\mathrm{as}}}{a_{01}}\right) n_{1} .
\end{aligned}
$$

If we ignore the $x^{2}$ and thus the $\tau^{4}$ terms in the foregoing equations by setting $A^{\prime}=0$, we find

$$
\tau_{\text {linear }}=\left(\frac{-C^{\prime}}{B^{\prime}}\right)^{1 / 2} \text {. }
$$


Regarding the \pm sign choice in Eq. (42), we note that $B^{\prime}$ is negative and $C^{\prime}$ is positive. If $A^{\prime}$ is positive, we must choose the + sign because it gives the correct limit given in Eq. (45) as $A^{\prime}$ goes to zero. If $A^{\prime}$ is negative, we must choose the - sign to avoid taking the square root of a negative number in Eq. (42). In Eq. (43), all expressions in round parentheses are positive, except that $\left(1 / D_{\text {ef } 1}-1 / D_{\mathrm{df} 1}\right)$ is negative for $\mathrm{N}_{2}$ and $\mathrm{CO}_{2}$ diluent gases. Thus $A^{\prime}$ is negative for $\mathrm{He}$, but for $\mathrm{N}_{2}$ and $\mathrm{CO}_{2}$ diluent gases the sign of $A^{\prime}$ depends on which term dominates in Eq. (43).

Results for the ternary gas systems are displayed in Table 2 below. There is a systematic decrease in the calculated tortuosity as the diluent concentration decreases, except for our calculations for He. Also, there is a systematic increase in calculated tortuosity as diluent molecular mass increases. In theory, the tortuosity should be independent of such experimental conditions. Although the reasons for these trends are not known, we do obtain the expected result that the $\tau$ values based on the three diluents approach each other as diluent concentration decreases.

The linear approximation to $\tau$ is accurate within a few percent. The $\tau$ values calculated by JV (the square roots of the " $\tau_{\mathrm{JV}}$ " values reported in their paper, which are really "tortuosity factors") are larger than ours for the light He diluent gas, but comparable to ours for the heavy $\mathrm{N}_{2}$ and $\mathrm{CO}_{2}$ diluent gases. The variation in our " $\tau_{\text {exact }}$ " values, and the differences between our " $\tau_{\text {exact }}$ " values and the corresponding $\left(T_{\mathrm{JV}}\right)^{1 / 2}$ values, is greater than for the binary systems presented in Table 1. Still, all of our $\tau_{\text {exact }}$ values, centered near 2.3 and ranging from about 1.7 to 3.3, are physically reasonable and provide confidence that JV's experimental method is valid for finding tortuosity.

Plots of gas concentrations across the anode for four ternary systems under anode limiting current conditions appear in Fig. 3. All show significant increases in total gas concentration $n$ (and thus in total pressure $P$ ) across the anode. All show decrease in diluent concentration going across the anode, because collisions with the heavier outflowing $\mathrm{H}_{2} \mathrm{O}$ molecules have more effect than those of the lighter inflowing $\mathrm{H}_{2}$ molecules, even though the $\mathrm{H}_{2} \mathrm{O}$ and $\mathrm{H}_{2}$ molecular flow rates are the same. The exponential curvature of the diluent concentrations is small but noticeable, whereas the quadratic and exponential corrections to the linear $x$ dependences of fuel and exhaust concentrations appear insignificant.

\section{Conclusions}

Based on all the above results, we consider that the tortuosity for the Jiang and Virkar apparatus [1] analyzed herein is $2.3 \pm 0.6$. This is fairly consistent with the range of 2.5-3 found by Williford et al. [3] for modern SOFC materials, and is also within the 1-3 range that seems most physically reasonable, 1 being the limit for an extremely open and probably impractical electrode design. The apparent disagreement between our values and the tortuosity values reported by Jiang and Virkar lies in their reporting as tortuosity what is often called the tortuosity factor, which is the square of the tortuosity [2] so we can say that their actual values and ours and those of Williford et al. are in general agreement and are physically reasonable.

The more exact method presented here for calculating tortuosity, or alternatively for calculating concentration drops across the electrode if tortuosity is known, is simple enough to justify its use. Knowing concentrations at the electrode-electrolyte interfaces is essential for calculating the concentration polarization term in the voltage $v s$. current density expression. Conversely, our expressions for activation and concentration polarizations based on opposing current flows at each electrode interface with the electrolyte presented herein and derived elsewhere [8] allow us to determine the tortuosity more accurately. Such voltage calculations, together with calculations of pressure drops across electrodes and of tortuosity as presented above, provide tools for predicting performance of new SOFC designs and for analyzing performance of existing designs, both in the fuel cell and electrolysis operation modes.

\section{Acknowledgements}

Helpful discussions with Anil Virkar and Stephen Sofie are gratefully acknowledged. This work was supported by DOE Award No. DE-AC06-76RL01830 as a subcontract from Battelle Memorial Institute and Pacific Northwest National Laboratories.

\section{References}

[1] Y. Jiang, A.V. Virkar, J. Electrochem. Soc. 150 (2003) A942-A951.

[2] N. Epstein, Chem. Eng. Sci. 44 (1989) 777-779.

[3] R.E. Williford, L.A. Chick, G.D. Maupin, S.P. Simmer, J.W. Stevenson, J. Electrochem. Soc. 150 (2003) A1067-A1072.

[4] R.S. Gemmen, J. Trembly, J. Power Sources 161 (2006) 1084-1095.

[5] V.H. Schmidt, C.-L. Tsai, L.M. Lediaev, in: N.P. Bansal (Ed.), Advances in Solid Oxide Fuel Cells III, Wiley, Hoboken, NJ, 2008, pp. 129-140.

[6] F. Reif, Fundamentals of Statistical and Thermal Physics, McGraw-Hill, New York, 1965, pp. 273-287.

[7] T.K. Sherwood, R.L. Pigford, C.R. Wilke, Mass Transfer, McGraw-Hill, New York, 1975.

[8] V.H. Schmidt, Dynamic first-principles molecular-scale model for solid oxide fuel cells, in: ECS Transactions, vol. 6, issue 21, Design of Electrode Structures, E. De Castro, L. Lipp, and D. Mah (Eds.), 2008, pp. 11-24. 